\title{
Diesel Exhaust
}

National Cancer Institute

\section{Source}

National Cancer Institute. Diesel Exhaust. NCI Thesaurus. Code C113679.

The airborne components that result from the combustion of diesel fuel. It includes both gaseous products of complete and incomplete combustion and particulates. 